\title{
Young patients with myocardial infarction - particularities of cardiovascular rehabilitation
}

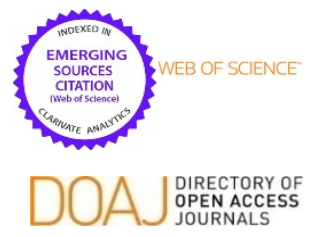

\author{
Ovidiu Anchidin', Dana Pop ${ }^{2}$
}

Corresponding author: Ovidiu Anchidin, E-mail: anchidin ovidiu@yahoo.com,

\section{Heart Institute "N. Stancioiu", Cluj-Napoca, Romania}

2. "Iuliu Hatieganu" University of Medicine and Pharmacy, Cluj-Napoca, Romania

3. Rehabilitation Hospital, Cluj-Napoca, Romania

\section{Abstract}

Acute coronary syndromes (ACS) are one of the main causes of morbidity and mortality in the world. It affects people in the middle and late-life, but the biggest psycho-social and financial impact is among the active young population. After the acute event they have to return to work and to their families for which they represent the principal support. Besides medication and prompt revascularization therapies, cardiac rehabilitation (CR) has an important role in regaining most of the initial physical and psychological state. Despite the proved benefit of the CR, referral and participation rate is very low, suggesting that there is more to do in this old but very underappreciated field.

Key words: acute coronary syndromes, myocardial infarction, young, cardiac rehabilitation, secondary prevention,

\section{Introduction}

Cardiovascular diseases (CVD) continue to be the main cause of mortality worldwide and represent almost a quarter of all diseases in Europe, while the costs involved for their treatment are considerable (1). Within 5 years of acute myocardial infarction (MI), about $15 \%$ of men and $22 \%$ of women aged up to 65 years will have a recurrence (2). Hence the importance of secondary prevention. In addition to the initiated treatment, lifestyle changing and cardiac rehabilitation (CR) are methods that have undeniably proved their efficacy. These consist of long-duration programs involving medical visits, prescription of physical exercise, modification of risk factors, education and counseling (3). Up to $90 \%$ of coronary disease cases occur in the context of well-known associated risk factors, among which smoking, sedentary lifestyle and inadequate diet stand out (4). The benefits of CR are well-established, and international guidelines recommend routine $\mathrm{CR}$ in standard treatment after MI. Thus, AHA/ACC guidelines for the treatment of patients with acute MI with ST elevation present it as a class I recommendation, while according to ESC guidelines, this is a class IIa recommendation $(5,6)$. Exercise can be considered a preventive method, a "pill" that should be administered daily due to its beneficial effects (7). Young patients who have suffered an acute coronary event represent a special class in that they are most often extremely active persons who are at a delicate time of their life. They have stressful tasks at work, many familial responsibilities, small

children and most frequently also parents in their care (8).

\section{History}

Recommendations related to $\mathrm{CR}$ have radically changed over the past decades. Initially, in the 1940's, patients who suffered a MI were advised to completely avoid physical exercise during convalescence, limiting themselves to rest in bed and subsequently in an armchair. In the 1950's, short daily walks for 3-5 minutes were recommended and only in the 1960's the first structured CR protocols were recommended at days after the acute event, through more sustained regular physical exercise (3). In Europe, the first physical exercise recommendations after a cardiac event were those of the German physician Max Oertel in 1885. His principles were applied later, in the 1950's, by Peter Beckman, and the first official CR program was launched in the 1970's in Hamburg, Germany (9). The first CR working group was created in 1982, as part of the European Society of Cardiology, and in 2004, the European Association for Cardiovascular Prevention and Rehabilitation (EACVPR) was founded (9). In the 60's, the first preventive cardiology and rehabilitation centers emerged in Romania, initially in Bucharest, under the leadership of Ioan Orha who also elaborated the first recommendations in the field, and subsequently, in other Romanian cities: Iași, Cluj-Napoca, Târgu Mureș, Timișoara and Covasna (10-12). 


\section{Evidence}

The first clear evidence regarding the efficacy of CR was provided by systematic reviews and metaanalyses published more than 20 years ago. These showed a $20-25 \%$ reduction of all-cause and cardiovascular mortality through the analysis of 22 randomized trials with a number of over 4300 patients. In the meantime, several improved versions were published (3). One of them was aimed at evaluating the effects of CR in post-MI patients who attended a standard program for at least 2 weeks, compared to those who did not benefit from such interventions. Thus, 34 randomized clinical studies with more than 6000 patients were selected. A diminution of MI recurrence risk (OR $0.53,95 \% \mathrm{CI}$ $0.38-0.76)$, a reduction of cardiac-cause mortality (OR 0.64, 95\% CI 0.46-0.88), as well as overall mortality (OR $0.74,95 \%$ CI $0.58-0.95$ ) were shown (13). Beneficial effects were also obtained on the incidence of cardiovascular RF: smoking, blood pressure (BP), body mass index (BMI), and plasma lipid profile. Besides the fact that even short period of CR was shown to have a positive impact, this persisted beyond the time of active period.

A series of imaging studies have shown an improvement of myocardial perfusion in patients who attended a supervised post-MI program of physical exercise, showing that the positive effect is not related only due to the peripheral physiological adaptation but to the afore development of collateral circulation also. Gunning et al. demonstrated a decrease in the perfusion defect using scintigraphy, as well as a reduction in the severity of the defect in patients enrolled in a 6-week CR protocol, consisting of 20minute exercise to a target heart rate between $60-80 \%$ of age-related maximum (14). Another study conducted by Lee et al. showed that a 3-month CR program carried out at an effort intensity determining a consumption of $55-70 \%$ of $\mathrm{VO} 2$ max led to an improvement of the myocardial perfusion reserve (MRI-quantified) by $25 \%$ in the infarcted area $(p<0.05)$ and by $30 \%$ in the adjacent myocardium $(p<0.01)$, with a consecutive increase in exercise capacity (15). Regarding the patients with left ventricular dysfunction (ultrasound-based), Sadeghi et al. revealed that those who attended a physical rehabilitation program for 8 weeks had a clear improvement in kinetics, with an increase in the left ventricular ejection fraction from $45.14 \% \pm 5.77 \%$ to
$50.44 \% \pm 8.70 \%(\mathrm{p}<0.001)(16)$.

A recently published systematic Cochrane metaanalysis shows the most important evidence of efficacy of physical exercise-based CR in terms of mortality, morbidity and quality of life in patients with ischemic heart disease (17). The meta-analysis comprised 63 studies published until July 2014, found in Cochrane Central Register of Controlled Trials, MEDLINE, EMBASE, CINAHL and Science Citation Index Expanded databases. These included almost 15,000 patients, with more than $80 \%$ post-MI patients, the rest being in the coronary revascularization group. The mean follow-up period was 12 months, and most of the studies had small size samples (between 28 and 2304 patients, on average 126 patients/study). A significant reduction in cardiovascular-cause mortality (RR $0.74,95 \%$ CI 0.64-0.86), in the rate of hospitalization (RR 0.82 , 95\% CI 0.70-0.96) as well as an improved healthrelated quality of life (HRQL) was observed, especially in younger men, but there were no differences regarding overall mortality (RR 0.96, 95\% CI 0.88-1.04), the rate of MI (RR 0.90, 95\% CI $0.79-1.07)$ or the rate of surgical coronary revascularization (RR 0.96, 95\% CI 0.80-1.16), and percutaneous coronary revascularization, respectively (RR $0.85,95 \%$ CI $0.70-1.04$ ) (17). The analysis of the 20 studies that assessed quality of life demonstrated beneficial effects of CR in this regard, while other studies showed a favorable costeffectiveness ratio $(18,19)$.

A Scandinavian trial investigated the impact of a 5year intensive lifestyle modification which included physical exercise, smoking cessation, dietary advice and stress management in younger women with cardio-vascular disease (CVD) and demonstrated a reduction in the presentation to the emergency department, in work absenteeism and positive effect on quality of life and public finances (20).

Pouche et al. published similar results regarding the impact of CR on mortality. Participants in the 2005 French FAST MI registry were followed up over a 5year period for the impact of the CR program on survival (21). Although the rate of prescription of CR was low $(22 \%)$, there was a benefit particularly in the reduction of mortality (by approximately $25 \%$ ), due to better control of RF, improved functional capacity and better patient compliance with treatment (21). After multivariate analysis, the effect was different in certain subpopulations. Young patients (aged less 
than 60 years) had a $66 \%$ reduction of mortality. Also, men and patients with MI without ST segment elevation benefited more from CR programs.

The efficacy of CR programs depends on certain standards that must be implemented and respected. Hence the need for programs for monitoring and quantifying the application of CR protocols. The first program of this type in Europe was Carinex Survey in 2002 (22), then ECRIS (European Cardiac Rehabilitation Inventory Survey) in 2008 (23). These provided information about the structure, legislation, financing and implementation of $\mathrm{CR}$ programs at national level. However, they did not provide clinical and efficacy data. The latest European project that was materialized was EuroCaReD (European Cardiac Rehabilitation Registry and Database), which was aimed at analyzing current CR practice in Europe based on an electronic data collection system (1). Data provided by 12 European countries, including Romania, regarding patients' basic characteristics, $\mathrm{CR}$ indications, treatment and evolution were recorded. The most frequent risk factors: hyperlipidemia, hypertension, sedentary lifestyle and smoking were identified. The most frequent indications of $\mathrm{CR}$ were acute coronary syndrome, post-PCI state electively and post-cardiac surgery state, and only $15 \%$ of patients did not complete the program. At least half of the patients benefited from $\mathrm{CR}$, with an improvement in exercise capacity by at least $25 \mathrm{~W}$. These were mainly young people aged less than 50 years, STEMI and post-surgery patients. Extremely heterogeneous findings were observed regarding the content, duration and place of CR. In Central and Eastern European countries, CR is initiated in hospital, and total duration is shorter, while evidence of efficacy provided by the literature is based on longer duration programs involving more sessions (1).

\section{CR. Definition and protocol}

The most accurate definition was launched by the British Association for Cardiovascular Prevention and Rehabilitation (BACPR): "The coordinated sum of activities required to influence favorably the underlying cause of cardiovascular disease, as well as to provide the best possible physical, mental and social conditions, so that the patients may, by their own efforts, preserve or resume optimal functioning in their community and through improved health behavior, slow or reverse progression of disease" (17). The current concept of CR comprises that it should be started immediately after the acute event or following the interventional procedure. Once started it should be continuous, staged and individualized depending on the patient preference and tolerance (24).

In the initial ECRIS study conducted by EACPR (European Association for Cardiovascular Prevention and Rehabilitation), CR was structured into three stages (23). The first two take place in the acute phase and are mandatory, preparing the patient for the outof-hospital activity.

\subsection{First stage (early in-hospital rehabilitation)}

After the pharmacotherapy initiation and the revascularization procedure, the patient is immobilized for 12-48 hours, depending on the clinical situation. He is informed about the disease, the severity of it and he is counselled for anxiety reduction and mental support. At the same time, psychosocial factors must be identified, which are extremely important in the case of young patients in particular, who are often exposed to stress at their workplace.

The inpatient performs mild exercise in order to recover minimum exercise capacity, required for daily routine activities. At first, this was recommended daily over 1-2 weeks, but with the successful implementation of revascularization procedures, it was minimized, due to immediate recovery of cardiac function (25).

In this situation, the importance of this stage resides in completely safe testing of patient's exercise capacity. Reassuring that the patient is stabilized doing minor in-room activities for itself, exercise of gradually increased intensity is initiated under physiotherapist supervision after 48 hours.

Also, at this stage, both the patient and the patient's family should be informed about the risk factors that have caused acute coronary syndrome and about the importance of cardiovascular prevention medication (statins, converting-enzyme inhibitors/sartans, antiplatelet drugs and beta-blockers).

\subsection{Second stage}

The proper rehabilitation is initiated 10-20 days after infarction, depending on the initial evolution of the disease, the treatment applied and the patient's exercise tolerance. This is recommended to be initiated in a specialized rehabilitation center, in collaboration with a team of kinesiotherapists, psychologists and dieticians, and can last up to 16 weeks. It consists of carefully monitored physical and 
psycho-educational activities (25).

The program starts with the performing of a standardized exercise test that will assess the patient's exercise capacity, residual myocardial ischemia and the presence of rhythm or conduction disorders. Patients with reduced exercise capacity (less than 5 METs) and/or exertional heart failure events will attend a moderate exercise program and will be carefully monitored in a dedicated residential or outpatient center. Patients with good tolerance will perform a physical activity program including at least 36 sessions (between 3 and 5 per week) with the aim of improving exercise capacity by $25-30 \%$ or reaching the threshold of 7 METs (25-27). On the other hand, the recent European Register on Cardio rehabilitation Euro Ca Re D defines successful CR as an increase in the exercise capacity by more than $25 \mathrm{~W}$, a target reached by only $58 \%$ of patients, particularly young and/or active patients (1). Physical exercises, particularly dynamic, on the cycle ergometer, but also static, by light weight lifting, will be performed. The intensity of training can be rigorously estimated initially using the cardiopulmonary exercise test (as metabolic equivalents - METs or percentage of VO2max), but most frequently, it is estimated based on the heart rate reached, which should not exceed $70 \%$ and $85 \%$ of the maximum heart rate in patients with low tolerance and trained subjects, respectively. This stage ends when the exercise test evidences an exercise capacity of 7 METs; otherwise, it is prolonged precisely in order to reach this threshold (25-27).

\subsection{Third stage}

This stage should be long-term, ideally lasting throughout the whole life, and is a basic component of patient's lifestyle changing. It consists of maintaining the previously obtained exercise capacity and is carried out individually and without supervision. It corresponds to maintenance physical activity recommended for healthy persons and consists of performing exercises for at least 30 minutes, ideally 60 minutes daily, 5 days a week (25). These may vary depending on the patient's ability and preference: from gardening, brisk walking, static cycling to higher intensity practice of team sports. A particular form of residential rehabilitation that can be applied in Romania is the one used in the Hospital of Cardiovascular Diseases Covasna, which is unique in Europe due mainly to the special climate conditions
(10-12).

\section{Conclusions}

Enrollment of young patients with a history of myocardial infarction in cardiovascular rehabilitation programs is beneficial, being accompanied by an increase in exercise capacity, as well as by a reduction in the rate of adverse cardiovascular events, contributing to their rapid socio-professional reintegration. In this context, implementing nonpharmacological and pharmacological secondary prevention measures will also be possible.

\section{References}

1. Benzer W, Rauch B, Schmid JP, et al. Exercisebased cardiac rehabilitation in twelve European countries results of the European cardiac rehabilitation registry. International Journal of cardiology. 2017;228:58-67.

2. Menezes AR, Lavie CJ, Milani RV. Cardiac rehabilitation in the United States. Progress in cardiovascular diseases. 2014;56:522-529.

3. Taylor RS, Zwisler AD. New concepts in cardiac rehabilitation and secondary prevention after myocardial infarction. In: Morrow DA, editor. Myocardial Infarction. 1st Ed.St.Louis, Missouri: Elsevier; 2017.

4. Sandesara PB, Lambert CT, Gordon NF et al. Cardiac rehabilitation and risk reduction. J. Am. Coll. Cardiol.2015;65:389-395.

5. Kushner FG, Hand M, Smith SC Jr, King SB 3rd, Anderson JL, Antman EM, et al. ACC/AHA guidelines for the management of patients with ST-elevation myocardial infarction (updating the 2004 guideline and 2007 focused update) and ACC/AHA/SCAI guidelines on percutaneous coronary intervention (updating the 2005 guideline and 2007 focused update) a report of the American College of Cardiology Foundation/American Heart Association Task Force on Practice Guidelines. J. Am. Coll. Cardiol.2009;5: 2205-2241.

6. Steg PG, James SK, Atar D, Badano LP, Blömstrom-Lundqvist C, Borger MA et al. ESC Guidelines for the management of acute myocardial infarction in patients presenting with ST-segment elevation, Eur. Heart J.2012;33:2569-2619.

7. Fletcher GF, Ades PA, Kligfield P, et al. Exercise standards for testing and training: a scientific statement from the American Heart Association. Circulation. 2013;128:873-934. 
8. Andersson EK, Borglin G, Willman A. The experience of younger adults following myocardial infarction. Qualitative health Research. 2013; 23(6):762-772.

9. Humphrey R, Guazzi M, Niebauer J. Cardiac rehabilitation in Europe. Prog Cardiovasc Dis. 2014;56(5):551-6.

10. Zdrenghea D, Branea I. Recuperarea bolnavilor cardiovasculari. Ed. Clusium, Cluj-Napoca, 1995.

11. Orha I, Apetrei E. Antrenamentul fizic la bolnavii cu infarct miocardic (Nota 1). Medicină Internă, București, 1975;2:73-80.

12. Mitu F, Roca IC, Roca M. Reabilitarea medicală a pacientului postinfarct miocardic. Viața Medicală 2016;46:6-7.

13. Lawler PR, Filion KB, Eisenberg MJ: Efficacy of exercise-based cardiac rehabilitation postmyocardial infarction: a systematic review and meta-analysis of randomized controlled trials. Am Heart J.2011;162:571-584.e2.

14. Gunning MG, Walker J, Eastick S et al. Exercise training following myocardial infarction improves myocardial perfusion assessed by thallium-201 scintigraphy. Int J Cardiol. 2002;84:233-239.

15. Lee BC, Chen SY, Hsu HC. Effect of cardiac rehabilitation on myocardial perfusion reserve in postinfarction patients. Am J Cardiol. 2008;101(10):1395-402.

16. Sadeghi M, Garakyaraghi M, Khosravi M. The impacts of cardiac rehabilitation program on echocardiographic parameters in coronary artery disease patients with left ventricular dysfunction. Cardiol Res Pract. 2013;2013:201713.

17. Anderson L, Thompson DR, Oldridge $\mathrm{N}$ et al.: Exercise-based cardiac rehabilitation for coronary heart disease: Cochrane systematic review and meta-analysis. J Am Coll Cardiol. 67:1-12 2016

18. Briffa TG, Eckermann SD, Griffiths AD et al. Cost-effectiveness of rehabilitation after an acute coronary event: a randomised controlled trial. Medical Journal of Australia. 2005;183:450-5.

19. Maddison R, Pfaeffli L, Whittaker R et al. A mobile phone intervention increases physical activity in people with cardiovascular disease: Results from the HEART randomized controlled trial. European Journal of Preventive Cardiology. 2014;22(6): 701-9.

20. Andersson A, Sundel KL, Undén AL, SchenckGustafsson K, Eriksson I. A five-year rehabilitation programme for younger women after a coronary event reduces the need for hospital care. Scand J Public Health. 2010 Aug;38(6):566-73.

21. Pouche M, Ruidavets JB, Ferrières J et al. Cardiac rehabilitation and 5-year mortality after acute coronary syndromes: The 2005 French FAST-MI study. Arch Cardiovasc Dis. 2016;109(3):178-87.

22. Vanhees L, McGee HM, Dugmore LD, Schepers D, van Daele P. A representative study of cardiac rehabilitation activities in European Union Member States. J. Cardpulm. Rehabil. 2002;22:264-272.

23. . Bjarnason-Wehrens B, McGee H, Zwisler AD, Piepoli MF, Benzer W, Schmid JP et al. Cardiac rehabilitation in Europe: results from theEuropean Cardiac Rehabilitation Inventory Survey, Eur. J. Cardiovasc. Prev. Rehabil. (2010);17:410-418.

24. Piotrowicz R, Wolszakiewicz J. Cardiac rehabilitation following myocardial infarction. Cardiol J.2008;15:481-7.

25. Pop D, Zdrenghea D, Rosu R et al. Recuperarea cardiovasculară: manual pentru studentii de la programul de studiu Balneofiziokinetoterapie și recuperare medicală. Cluj-Napoca: Editura Medicală Universitară"Iuliu Hațieganu", 2016.

26. .Fletcher GF, Ades PA, Kligfield P et al. Exercise standards for testing and training: a scientific statement from the American Heart Association. Circulation. 2013;128(8):873-934.

27. Piepoli MF, Corrà U, Adamopoulos $\mathrm{S}$ et al. Secondary prevention in the clinical management of patients with cardiovascular diseases. Core components, standards and outcome measures for referral and delivery: a policy statement from the cardiac rehabilitation section of the European Association for Cardiovascular Prevention \& Rehabilitation. Endorsed by the Committee for Practice Guidelines of the European Society of Cardiology. Eur J Prev Cardiol. 2014;21(6):66481. 